\title{
The role of test procedure in linguistic integration studies
}

\author{
RICHARD A. GRIGGS and DONALD M. KEEN \\ University of Florida, Guinesville, Florida 32611
}

\begin{abstract}
The effects of type of recognition test procedure were studied in a Bransford and Franks (1971) integration paradigm. Subjects received a two-alternative forced-choice recognition test or a modified "forced-choice" test in which all the sentences for each idea set were presented at once and the "old" sentences had to be identified. Contrary to the usual Bransford and Franks results, in which a yes-no one-sentence-at-a-time recognition procedure is employed, the ability to discriminate "old" sentences from "new" sentences was clearly observed. A bias for selecting more complex sentences, however, was found for the modified "forced-choice" procedure. A prototype learning model is described to account for these results and previous data.
\end{abstract}

In a seminal study on the abstraction of linguistic ideas, Bransford and Franks (1971) found results which suggested that, when subjects heard a set of semantically related sentences, they appeared to integrate the information communicated by the set of sentences to construct a more "holistic semantic structure." The semantic structures studied by Bransford and Franks were ideas underlying complex embedded sentences. Each complex idea consisted of four basic propositions. For example, the complex idea "the rock which rolled down the mountain crushed the tiny hut at the edge of the woods" can be broken down into four simple propositions: "the rock rolled down the mountain," "the rock crushed the hut," "the hut was tiny," and "the hut was at the edge of the woods." The complete idea is referred to as a FOUR and the four simple propositions as ONES. ONES can also be combined to form TWOS and THREES, meaningful combinations of two or three simple propositions. The entire set of ONES, TWOS, THREES, and the FOUR is referred to as an idea set.

Bransford and Franks (1971) employed four such idea sets. Their abstraction paradigm consisted of an incidental acquisition phase in which subjects were presented with six sentences of varying complexity from each idea set, followed by an unexpected recognition test. On the test, subjects were presented with another set of sentences one at a time and asked to indicate which of the sentences they had heard before and which they had not. Test sentences were of three general types: "olds" (sentences presented in acquisi-

This research was presented at the annual meeting of the Southeastern Psychological Association, Hollywood, Florida, May 1977. The authors wish to thank John Anderson and an anonymous reviewer for their comments and suggestions on an earlier version of this paper. Requests for reprints should be sent to Richard A. Griggs, Department of Psychology, University of Florida, Gainesville, Florida 32611. tion), "news" (sentences in one of the idea sets but not presented in acquisition), and "noncases" (sentences containing information present in the four idea sets but not derivable from only one idea set or sentences formed by changing the modification role of one of the propositions in an idea set).

Bransford and Franks (1971) found that subjects were unable to differentiate olds and news; subjects could, however, accurately recognize noncases as having not been presented, and recognition performance was a positive linear function of sentence complexity. Recognition ratings were ordered FOURS $>$ THREES $>$ TWOS $>$ ONES. Bransford and Franks interpreted these results to mean that subjects did not store the individual sentences, but rather integrated the information given in the sentences for each idea set to construct holistic semantic ideas and stored the ideas in memory. Recognition responses were a function of these holistic ideas. Olds and news were both recognized but not differentiaated from each other, because both were derivable from the complete ideas. Recognition reflected the degree to which a sentence exhausted the complete idea involved. Replication of these results has been reported several times (e.g., Schwartz \& Witherspoon, 1974; Singer, 1973) and the major effects of complexity and the inability to distinguish old from new items also occur with recall tests (Cofer, 1973; Griggs, 1974).

Throughout the above studies, however, two major variables are held constant. Acquisition occurs in an incidental task environment, and the relatively insensitive yes-no recognition procedure is employed rather than a forced-choice procedure. The possibility of a strong effect of an intentional acquisition phase, that is, one in which subjects are told a recognition test will be given following acquisition, is intuitively high. White (1974) replicated Bransford and Franks (1971) using two groups; one group received incidental acquisition instructions, as in the original Bransford and Franks 
experiment, and the other group was told to remember the sentences for a later recognition test. The incidental condition produced the typical Bransford and Franks results. In the intentional condition there was a lesser tendency to abstract general ideas; FOURS received lower ratings than THREES. Additionally, inspection of the hit and false alarm rates showed a significant ability to distinguish olds and news. It appears that, with intentional instructions, of the two primary Bransford and Franks results, only a weakened complexity effect remains.

Anderson and Bower (1973, pp. 351-352) reported a study in which they employed both an intentional acquisition phase and a forced-choice testing procedure. Anderson and Bower argued that the subject's inability to discriminate olds from news was a result of the yes-no recognition procedure and that a forced-choice procedure caused more discriminative processing of the test sentences. The results of this experiment revealed no complexity effect and an ability to discriminate olds and news. Comparing this with White's (1974) study, the complete disappearance of the complexity effect appears to be the unique result of the forcedchoice procedure. This occurred even though the standard version of the two-alternative forced-choice procedure was not employed. Instead, subjects were presented the 12 sentences from each idea set and asked to identify the six acquisition sentences.

Based on these results and their postulated model of memory (HAM), Anderson and Bower proposed two processing strategies that subjects might employ in a Bransford and Franks recognition test. These strategies are dependent upon the recognition test procedure. According to Anderson and Bower, the memory representation of the six acquisition sentences for each idea set is a propositional binary tree of the simplest propositions (ONES), each with contextual taggings containing information about the context of their acquisition. Each of the four simple propositions occurs three times during acquisition and therefore is attached to three contexts. One strategy for processing this memory representation is called "context counting." Recognition rating depends upon the average number of contexts evoked by the test sentence. The average number of contexts activated will increase with the number of simple propositions contained in the test sentence. It is also the case that the average number of contexts activated will be the same for old sentences as for new sentences. Therefore, such a strategy should result in findings like those of Bransford and Franks (1971).

Another possible strategy is a context-searching strategy. For this strategy, the subject must examine all possible contextual linkages of all the simple propositions in the test sentence, see if there are any intersections, verify that the intersecting contexts are attached to all the target propositions, and, finally, make sure that no nontarget propositions lead to any of the intersecting contexts. This context-searching strategy would allow subjects to discriminate old from new and would, therefore, account for the Anderson and Bower forced-choice results.

Another explanation of the Bransford and Franks results was offered by Reitman and Bower (1973). They proposed a "learning" model in which particular information (individual sentences) is stored until the prototype (the complex idea) is built up, that is, learned. Once the prototype is abstracted, further particular information is no longer stored. Sentences presented after that are identified as the prototype plus some correction, for example, the prototype minus one proposition. Hence, stored particular information will explain the ability to discriminate some of the old information from the new. When a particular sentence is not stored, responding is based upon how much of the prototype it exhausts, producing a complexity effect. Reitman and Bower argued that the typical Bransford and Franks materials and procedures caused massive forgetting of the particular information and, thus, subjects are unable to differentiate old and new sentences.

The key factors in all of the above studies appear to be those mentioned earlier: intentional/incidental acquisition and type of test procedure. Different results occur with manipulation of these variables. The context strategies proposed by Anderson and Bower and the prototype learning model of Reitman and Bower can explain the available data. The present study attempted to provide some evidence necessary to choose between these explanations by investigating the combination of the above two variables that has not been studied, that is, incidental acquisition paired with a forcedchoice test procedure. An incidental acquisition paradigm, as in Bransford and Franks (1971), with both an ordinary two-alternative forced-choice testing procedure and the "forced-choice" procedure of Anderson and Bower (1973) was employed. Two versions of the latter procedure were investigated. In one version subjects were told how many old sentences there were and in the other version they were not. If subjects did not know exactly how many sentences were actually presented and had stored only the complex ideas, they might tend to recognize more than six sentences per idea set if not instructed how many to recognize.

The theoretical approaches discussed above make different predictions for the present experiment. Anderson and Bower would predict predominant use of the context-searching strategy because of the forcedchoice testing. Thus, an ability to discriminate olds and news should be observed in all conditions, but no complexity effects should be found. The prototype "learning" model would also predict an ability to dis- 
criminate olds and news in all the conditions, but also complexity effects in the two modified forced-choice conditions.

\section{METHOD}

\section{Subjects}

Fifty-two subjects in an introductory experimental psychology class at the University of Florida served as subjects. Subjects were randomly assigned to one of three groups, determin:d by the type of recognition test material given them. Nineteen subjects were placed in the two-alternative forced-choice (2A-FC) condition and the Anderson and Bower modified forced-choice condition with a numerical cue (FC-6), while 14 subjects were assigned to the Anderson and Bower type forced-choice condition with no numerical cue (FC).

\section{Materials}

The idea sets were taken from Katz (1973) and are very similar to those used by Bransford and Franks (1971). The four complex sentences used were: (1) The rock which rolled down the mountain crushed the tiny hut at the edge of the sea, (2) The ants in the kitchen ate the sweet jelly which was on the newspaper, (3) The barking dog chased the brown cat which jumped on the girl, and (4) The thunder crashing through the valley shook the tinkling bells in the corner. Two THREES, two TWOS, and two ONES were taken from each idea set to form the acquisition list. The sentences on the acquisition list were randomly ordered with the constraint that no two consecutive sentences were from the same idea set. Each subject was provided a lined blank sheet, numbered from 1 to 24 , on which to record answers during acquisition.

The recognition materials and their accompanying instructions were varied to form the three procedural conditions. The subjects in the $2 \mathrm{~A}-\mathrm{FC}$ condition each received a booklet with test instructions and, on each subsequent page, a single pair of sentences from the same idea set, one old and one new. All 12 sentences from each idea set were used on the test. Thus, there were six test pairs for each idea set. Each pair was randomly formed with the constraint that each sentence was of the same level, with the exception that for each idea set a new FOUR was paired with an old THREE. Subjects in this condition were instructed to check the old sentence on each page. Subjects in the FC and FC-6 conditions received recognition materials consisting of an instruction sheet and a random listing of the entire set for each of the four idea sets on separate pages. Only the instruction sheet differed for these two conditions. The FC- 6 instruction sheet told subjects that on each page (for each idea set) six of the sentences were old and six were new. The FC instruction sheet provided no such numerical information. For both conditions, subjects were instructed to indicate which sentences were old.

\section{Procedure}

All 52 subjects were run simultaneously in a normal classroom setting. Subjects were given the acquisition response sheets and informed that the experiment would involve a short-term memory task. A series of sentences (the acquisition list) was then read to the subjects. Each sentence was followed by an intervening counting task and then a question about the sentence. The trial by trial format was: (1) a sentence was read, (2) a card containing a number from two to five was shown, (3) the subjects counted backward aloud to one from that number, (4) an elliptical question was asked concerning the information in the sentence and the subject was given 5 sec to write the answer before the next trial began. The procedure was clearly explained to the subjects before the presentation of the acquisition list and some practice trials were included.

After completion of the acquisition phase, the subjects were told to place the response sheets aside and were then given the recognition material. Subjects were instructed to carefully read the instructions and then to proceed to the subsequent pages at their own pace. Both response sheets and test booklets were collected at the completion of the task.

\section{RESULTS}

Two probabilities, hit rate (the probability of correctly responding "old" to an old item) and false alarm rate (the probability of incorrectly responding "old" to a new item), were calculated for each subject for each level of sentence complexity and test procedure. These probabilities were used in a four-way SPF-p.qr analysis of variance (subjects nested in test type by level of sentence complexity by test type by probability measure) (Kirk, 1968, pp. 297-307).

One prior analysis was required in the $2 \mathrm{~A}-\mathrm{FC}$ condition. In this condition an old THREE could be paired with either a new THREE or a new FOUR. Consequently, two hit rates could be calculated for THREES. A two-tailed $t$ test between these two hit rates failed to reach significance $[\mathrm{t}(18)=1.31, \mathrm{p}>.15]$. Thus, the rates were combined in all subsequent analyses.

The four-way analysis of variance revealed a significant main effect for level of sentence complexity $[\mathrm{F}(2,98)=35.87, \mathrm{MSe}=.030, \mathrm{p}<.01]$, a main effect for probability measure $[\mathrm{F}(1,49)=47.88, \mathrm{MSe}=.037$, $\mathrm{p}<.01]$, and a significant Level of Sentence Complexity by Test Type interaction $[\mathrm{F}(4,98)=13.3$, $\mathrm{MSe}=.030, \mathrm{p}<.01]$. The three-way interaction, Level of Sentence Complexity by Test Type by Probability Measure, was significant at the .10 level $[F(4,98)=2.25$, $\mathrm{MSe}=.037, \mathrm{p}=.068]$. No other effects or interactions were significant using $p>.10$ as the rejection region.

To clarify the effects of sentence complexity, the mean probabilities for responding "old" for each level of sentence complexity by test type combination were computed. Multiple comparison analyses were performed using Tukey's h.s.d. statistic with the modification for an unweighted-means analysis (Kirk, 1968, pp. 276-278). No means were included in this analysis for FOURS since only the false alarm rate could be calculated. The means for ONES and TWOS for both FC (.25 and .57) and FC-6 (.35 and .51) test procedures were significantly different at the .01 level. The differences between means for TWOS and THREES for both these test procedures (.09 for FC and .07 for FC-6) failed to reach significance at the .05 level but are clearly in the direction predicted by Bransford and Franks (1971). Recognition probability is an increasing function of sentence complexity for both the FC and FC-6 procedures; there is a bias to select sentences with more propositions.

Due to the nature of the 2A-FC task in this experiment, the overall probability of responding "old" has to be .50 for each level of complexity. Hence, to further 
clarify the relationship of the three experimental factors, the minimum difference values for Tukey's h.s.d. statistic were calculated for the means within the three-way Level of Sentence Complexity by Test Type by Probability Measure interaction. These means are presented in Figure 1. A p value of .10 was employed, since $\mathrm{p}=$ .068 for the three-way interaction.

Testing for differences between hit and false alarm rates for each combination of complexity and test type provides information about the subjects' ability to discriminate old and new. For all three test procedures, the multiple-comparison procedure showed that hit rate and false alarm rate were significantly different for both ONES and TWOS. For the FC- 6 test procedure, this was also the case for THREES and, for the FC condition, the difference in probabilities for THREES was close to significance. Overall, there was a definite ability to differentiate old and new for all three test procedures (.56 vs. .42 for FC, .58 vs. .39 for FC-6, and .56 vs. .44 for $2 \mathrm{~A}-\mathrm{FC}$ ). This is confirmed by the fact that a significant main effect was observed for probability measure, but the Probability Measure by Test Type interaction was not significant.

In the FC and FC- 6 conditions the ability to discriminate old and new was found, but, at the same time, Bransford and Franks' bias to select more complex sentences was also observed. To further investigate the Reitman and Bower (1973) "learning" model for the present results, the response protocols for old sentences for each subject in both the FC and FC-6 conditions were checked. The proportion correct for old sentences that, according to such a learning model, would be stored and used to build the prototype and the proportion correct for old sentences presented after the prototype was supposedly built were calculated.

While the exact time of prototype completion for each subject and idea set was indeterminable in this experiment, the simple assumption of prototype learn-

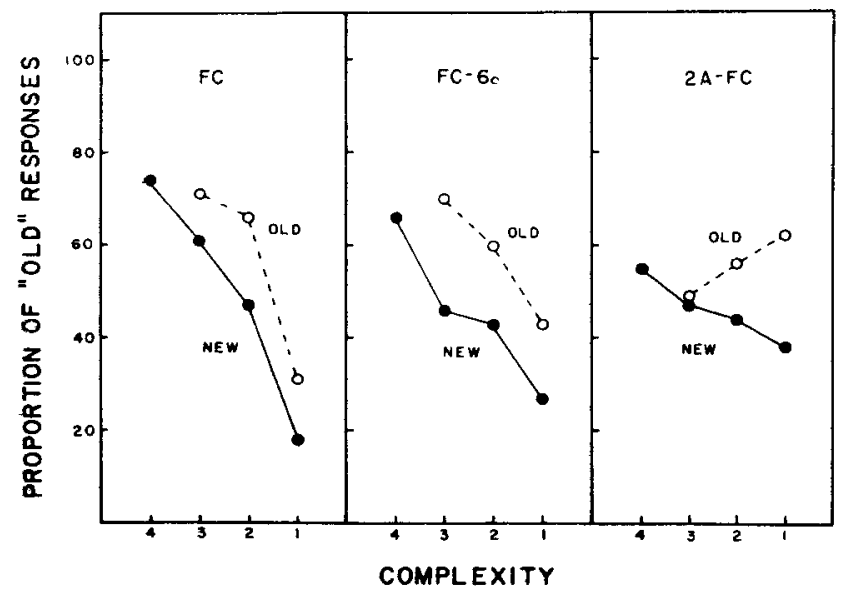

Figure 1. Proportion of "old" responses as a function of sentence complexity for both old and new sentences. ing as soon as sufficient information has been presented was testable. The proportions correct were computed using this assumption. Using one-tailed sign tests, no significant differences were observed in either condition. In the FC condition, only 6 of 14 subjects had a higher proportion correct on prior old sentences and, in the FC-6 condition, only 9 of 19 subjects did.

\section{DISCUSSION}

Clearly, the original explanation offered by Bransford and Franks (1971) must be altered. Even limiting acquisition to incidental methods does not produce the results predicted by a theory of general abstract knowledge only. The ability of subjects to distinguish olds and news for ONES and TWOS in the 2A-FC condition and all levels in the FC and FC- 6 conditions provides clear evidence for storage of some particular information. The data, however, are not predicted completely by either of the other proposed explanations. The complexity effect present in the FC and FC- 6 conditions is not predicted by the Anderson and Bower (1973) context strategies, and a strong learning assumption for the Reitman and Bower (1973) model is not supported. This assumption states that a sentence is stored if, and only if, any particular information contained in the sentence is not present in the current state of the prototype for the appropriate idea set.

Except for lack of support for the strong learning assumption, Reitman and Bower's prototype learning explanation can account for the present results. Therefore, consider a more realistic abstraction assumption for an incidental task, that is, that particular information is stored at random until the prototype is built up. This assumption will lead to the memory storage of the prototype plus a subset of the presented sentences. However, since no information concerning actual individual subject and idea set prototype completion times is available, this random learning assumption is not testable in the present experiment. If such completion times were available, the revised assumption would lead to a prediction similar to that for the strong learning assumption (i.e., subjects should be better on recognition of particular information prior to prototype completion than after).

Such a modified learning model can also fit the results of the studies discussed earlier. While this model easily accounts for the complexity effects observed by Bransford and Franks (1971), it would also predict a slight but definite ability to distinguish olds from news. Although theoretically present for all levels of complexity, any ability to recognize olds and news will be most apparent for ONES. Since the probability of an "old" response increases with complexity, any ability to distinguish olds and news for Levels TWO and THREE is confounded; that is, for TWOS and, especially, THREES, an "old" response is probably 
due to the complexity factor. This tendency is not present for ONES, and only recognition of the individual sentence should cause an "old" response. Reviewing the original Bransford and Franks (1971) results, they report a significantly higher recognition rating for old ONES than for new ONES. This is exactly what the modified learning model would predict.

Altering the acquisition instructions from incidental to intentional produces two effects (White, 1974). First, a clear ability to distinguish olds from news is present. This is easily handled by the proposed model with a different, but intuitive, abstraction assumption. Rather than assuming that learning of individual sentences is random and ceases as soon as the abstract prototype is completed, in an intentional learning situation it is assumed that the subject adopts a conscious strategy of attempting to learn all sentences. Thus, completion of the abstract FOUR does not halt the learning process. Clearly, this will produce a higher ability to distinguish olds from news. This ability should reveal itself primarily as a decrease in responding "old" to news. Since the complexity effect produces a high hit rate for THREES and FOURS already in White's study, it is unlikely that the hit rate will increase. The tendency to falsely recognize news caused by the complexity effect, however, will be counterbalanced by the increased learning of the individual sentences. A lower false alarm rate for TWOS, THREES, and FOURS should be produced. This effect appears to be present in the White (1974) data, although it is not tested. The slight complexity effect remaining for this study possibly reflects processing errors caused by memory load and conflicting processing decisions based on memory for individual sentences and exhaustion of the abstract prototype.

The addition of a forced-choice testing procedure, as in Anderson and Bower (1973), to an intentional acquisition task eliminates the complexity effect and again produces an ability to discriminate olds and news. The major effect of the forced-choice procedure is the alleviation of memory load and decision difficulties in the recognition task. Decisions can then be based upon the relative strength of individual sentences rather than upon an absolute yes-no criterion. Since these considerations apply to response processes and not to the structure of the representation, the results are compatible with the modified learning model.
The modified prototype learning explanation accounts for the general patterns observed in the da. 1 , but undoubtedly the explanation needs to be augmented and investigated further. ${ }^{1}$ The present data, however, strongly support the argument that particular information, that is, information about which individual sentences were presented, is stored in memory. When more sensitive test procedures are employed, subjects clearly demonstrate the ability to discriminate between old and new sentences. Contrary to Bransford and Franks (1971), it appears that both prototypical and particular information is stored.

\section{REFERENCES}

Anderson, J. R., \& Bower, G. H. Human associative memory. Washington, D.C: V. H. Winston, 1973.

Bransford, J. D., \& Franks, J. J. The abstraction of linguistic ideas. Cognitive Psychology, 1971, 2, 331-350.

Cofer. C. N. Constructive processes in memory. American Scientist, 1973, 61, 537-543.

GRIGGs. R. A. The recall of linguistic ideas. Journal of Experimental Psychology. 1974. 103, 807-809.

Katz, S. Role of instructions in abstraction of linguistic ideas. Joumal of Experimental Psychology, 1973. 98, 79-84.

KIRK. R. E. Experimental design: Procedures for the behavioral sciences. Belmont. Calif: Brooks/Cole, 1968.

Reitman. J. S.. \& Bower. G. H. Storage and later recognition of exemplars of concepts. Cognitive Psychology, 1973, 4, 194-206.

SChWARTz. S.. \& Witherspoon, K. D. Decision processing in memory: Factors influencing the storage and retrieval of linguistic and form identification. Bulletin of the Psychonomic Society, 1974, 4, 127-129.

SINGER, M. A replication of Bransford and Franks' (1971) "The abstraction of linguistic ideas". Bulletin of the Psychonomic Society. 1973. 1, 416-418.

W Hite, K. G. Abstracting ideas from sentences. New Zealand Psychologist, 1974, 4. 74-84.

\section{NOTE}

1. John Anderson has informed us that a model assuming subjects employ a mixture of the two Anderson and Bower context strategies will also account for the present results. Such a model would require precise assumptions of how task demands affect the usage of the particular strategies; these assumptions would seem difficult to derive. For this reason, the prototype learning model was preferred.

(Received for publication May 6, 1977; revision accepted September 9, 1977.) 